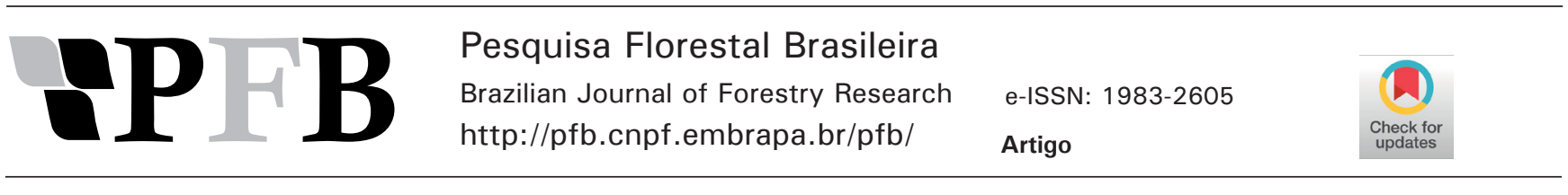

\title{
Crescimento de guanandi em sistemas agroflorestais e em plantio homogêneo em planície inundável
}

\author{
Antonio Carlos Pries Devide ${ }^{1}$ (i) , Cristina Maria de Castro ${ }^{1}$ (i) , Raul de Lucena Duarte Ribeiro ${ }^{+}{ }^{(\mathbb{D}}$, Antonio Carlos de Souza Abboud ${ }^{2}$ (i), \\ Marcos Gervasio Pereira ${ }^{2}$ (D) \\ ${ }^{1}$ Agência Paulista de Tecnologia dos Agronegócios, Avenida Antônio Pinheiro Junior, 4009, CEP 12400-970, Pindamonhangaba, SP, Brasil \\ ${ }^{2}$ Universidade Federal Rural do Rio de Janeiro, Instituto de Agronomia, BR 465, km 7, CEP 23897-000, Seropédica, RJ,Brasil \\ ${ }^{\dagger}$ In memoriam
}

"Autor correspondente:

antonio.devide@sp.gov.br

Termos para indexação:

Calophyllum brasiliense

Tolerância à inundação

Florestação

Index terms:

Calophyllum brasiliense

Flooding tolerance

Forestation

Histórico do artigo:

Recebido em 14/05/2019

Aprovado em 11/02/2021

Publicado em 29/08/2021
Resumo - O objetivo desse trabalho foi avaliar o crescimento de guanandi (Calophyllum brasiliense Cambess.), em planície inundável, em plantio homogêneo (controle) e em dois sistemas agroflorestais - SAFs (simples e biodiverso). O plantio de guanandi foi instalado no Vale do Paraíba, estado de São Paulo, em 2007. De 2011 a 2014 avaliou-se o crescimento de guanandi em um experimento em delineamento em blocos ao acaso, com oito repetições. Os SAFs consistiram do plantio de culturas anuais nas entrelinhas de guanandi. O SAF biodiverso foi acrescido de 16 espécies arbóreas, bananeiras (Musa sp.) e palmeira juçara (Euterpe edulis Mart.). As árvores de guanandi apresentaram similaridade morfológica no plantio homogêneo e nos sistemas agroflorestais, atingindo, em média, 5,40 m de altura total aos sete anos de idade. A taxa de crescimento relativo foi similar nos três tratamentos, com maiores valores atribuídos ao raio da copa $(2,59 \%)$ e à circunferência a $1,30 \mathrm{~m}$ do solo (1,86\%). Os sistemas agroflorestais são promissores, por aliar os benefícios da geração de renda e da restauração ecológica ao plantio de guanandi em planície de inundação.

\section{Comparative growth of guanandi in agroforestry systems and homogeneous stand in floodplain}

\begin{abstract}
The objective of this work was to evaluate the growth of guanandi (Calophyllum brasiliense Cambess.) in floodplain, as homogeneous planting (control) and as two agroforestry systems - AFS (simple and biodiverse). Guanandi was planted in the Paraíba Valley, State of São Paulo in 2007. From 2011 to 2014, guanandi growth was evaluated in a randomized block design experiment with eight replications. The AFS consisted of planting annual crops between guanandi lines. The biodiverse AFS was formed with 16 tree species, banana shrubs (Musa sp.) and juçara palm (Euterpe edulis Mart.). Guanandi presented morphological similarity in homogeneous planting and agroforestry systems and reached, on average, $5.40 \mathrm{~m}$ of total height at seven years of age. The relative growth rate was similar in the three treatments with higher values attributed to the canopy radius $(2.59 \%)$ and the circumference at $1.30 \mathrm{~m}$ above ground level $(1.86 \%)$. Agroforestry systems are promising to combine the benefits of income generation and ecological restoration in planting guanandi in floodplain.
\end{abstract}




\section{Introdução}

Cresce no Brasil o número de reflorestamentos com espécies nativas (Souza et al., 2018). O interesse no plantio de guanandi (Calophyllum brasiliense Cambess.) se deve pela qualidade de sua madeira (Carvalho, 2003; Durigan et al., 2012), que apresenta alta densidade. Suas árvores podem atingir de 20 a 30 $\mathrm{m}$ de altura com diâmetro do tronco de 40 a $60 \mathrm{~cm}$, podendo também ser usadas para captura de carbono da atmosfera (Petit \& Montagnini, 2006; Redondo-Brenes \& Montagnini, 2006).

A espécie da família Gutiferaceae é nativa do Brasil, ocorrendo em fitofisionomias da América Central, na Floresta Amazônica, em matas pantanosas nas depressões dos Cerrados (Oliveira \& Joly, 2010) até a costa Sudeste do Brasil na Mata Atlântica, onde os guanandizais naturais são muito raros, com exceção do litoral paranaense (Carvalho, 2003).

Por sua tolerância ao alagamento, o guanandi é amplamente utilizado na restauração ecológica de áreas inundáveis (Oliveira \& Joly, 2010; Durigan et al., 2012) e em solos ácidos com elevados teores de argila e baixos teores de nutrientes. Em áreas úmidas do Vale do Paraíba, entre os estados de São Paulo e Rio de Janeiro, a crescente ocupação do solo degrada as planícies de inundação e matas ciliares (Bittencourt \& Batista, 2009; Coelho et al., 2011; D’Orazio \& Catharino, 2013; DelRio et al., 2015). A renaturalização dessa paisagem, por meio da revegetação, pode auxiliar em reforço à laminação das cheias, evitando-se inundações em áreas ocupadas do entorno (Baptista \& Valcarcel, 2018). Entretanto, o plantio homogêneo de guanandi nesse compartimento da bacia hidrográfica é menos atrativo sob o ponto de vista econômico e social, pois as árvores levam muitos anos até o estádio de corte.

Em termos mundiais, a restauração ecológica combinando árvores em sistemas agroflorestais (SAFs) pode abranger bilhões de hectares com florestas plantadas ou em mosaico (Minnemeyer et al., 2011). Os SAFs podem unir os interesses da restauração ecológica e de geração de renda, por meio da produção de alimentos e produtos florestais, de maneira simultânea ou em sequência temporal (Coutinho et al., 2018; Devide et al., 2019). O uso de Calophyllum sp. em SAFs é amplamente reconhecido na Costa Rica (RedondoBrenes \& Montagnini, 2006), em ilhas do pacífico
(Friday \& Okano, 2006; Friday \& Ogoshi, 2011) e na Amazônia brasileira (Schroth et al., 2002).

O objetivo com esse trabalho foi avaliar o crescimento do guanandi em dois sistemas agroflorestais (simples e biodiverso) comparado ao plantio homogêneo (controle). A hipótese é de que em ao menos um SAF o crescimento do guanandi supere o plantio homogêneo.

\section{Material e métodos}

O estudo foi realizado em uma planície aluvial do Rio Paraíba do Sul com altitude média de $540 \mathrm{~m}$, em Pindamonhangaba, SP (22 $53^{\circ}$ S e $\left.45^{\circ} 23^{\prime} \mathrm{W}\right)$. O ecossistema de referência é a Floresta Semidecídua de várzea. O clima na região é caracterizado como Cwa subtropical de Köppen, com invernos secos, e temperaturas inferiores a $18{ }^{\circ} \mathrm{C}$, e verões quentes, com temperaturas superiores a $22^{\circ} \mathrm{C}$. A área experimental é contornada pelo ribeirão Capituba, que inundou com a alta precipitação pluvial na primavera-verão dos anos de 2011 (1.307 mm) e 2012 (1.497 mm) e interrompeu seu fluxo na seca extrema que ocorreu em 2013 $(1.158 \mathrm{~mm})$ e 2014 (619 $\mathrm{mm})$.

Os solos cultivados com guanandi foram classificados por Santos et al. (2013) como Planossolo háplico, Argissolo amarelo, Cambissolo flúvico e Gleissolo háplico, com valores médios de $288 \mathrm{~g} \mathrm{~kg}^{-1}$ de argila, $546 \mathrm{~g} \mathrm{~kg}^{-1}$ de areia, $166 \mathrm{~g} \mathrm{~kg}^{-1}$ de silte, alta densidade $\left(2,81 \mathrm{t} \mathrm{m}^{-3}\right)$ e drenagem deficiente. A fertilidade (Tabela 1) foi determinada, conforme Donagema et al. (2011). As amostras compostas foram formadas por quatro sub-amostras colhidas ao acaso nas entrelinhas do guanandi, com trado tubular, nas profundidades de 0-5, 5-10 e 10-20 cm, antes da implantação do experimento.

O guanandi foi plantado em 2007 em tabuleiros remanescentes do cultivo de arroz (Oryza sativa L.), no espaçamento $4 \mathrm{~m} \times 3 \mathrm{~m}$. As mudas foram dispostas em leiras elevadas a $30 \mathrm{~cm}$ de altura, preparadas com implemento de discos invertidos puxado com tração mecânica. As leiras foram usadas para que as raízes do guanandi explorassem um ambiente com maior oferta de oxigênio (Attanasio et al., 2015).

Em 2011 teve início a conversão agroflorestal, para tornar compatível a atividade econômica da exploração do guanandi com os interesses da restauração ecológica. Em setembro de 2011 implantou-se o experimento em 
delineamento em blocos ao acaso, para isolar os efeitos das variáveis edáficas, agrupando-se dois blocos em um mesmo tabuleiro para cada classe de solo. O crescimento do guanandi foi avaliado em três tratamentos: plantio homogêneo (controle), SAF simples (Figura 1A) e SAF biodiverso (Figura 1B), com oito repetições de cada tratamento em um total de 24 parcelas de $216 \mathrm{~m}^{2} \mathrm{com}$ quatro linhas de seis árvores de guanandi em cada linha de cada parcela.

Tabela 1. Constituição química inicial na camada superficial dos solos cultivados com guanandi em planície inundável, em Pindamonhangaba, SP.

Table 1. Initial chemical constitution in the superficial layer of the floodplain soils cultivated with guanandi in Pindamonhangaba, São Paulo State, Brazil.

\begin{tabular}{|c|c|c|c|c|c|c|c|c|c|c|}
\hline Prof & pH & $\mathbf{P}$ & $\mathbf{K}$ & $\mathrm{Ca}$ & Mg & Al & $\mathbf{H}+\mathbf{A l}$ & $\mathbf{S}$ & $\mathbf{T}$ & $\mathbf{V}$ \\
\hline $\mathrm{Cm}$ & \multicolumn{9}{|c|}{ 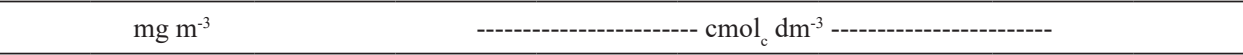 } & $\%$ \\
\hline 0 a 5 & 5,32 & 9,28 & 0,24 & 1,54 & 0,83 & 0,34 & 3,55 & 2,67 & 6,22 & 43,02 \\
\hline 5 a 10 & 5,30 & 8,09 & 0,21 & 1,36 & 0,83 & 0,40 & 3,48 & 2,46 & 5,94 & 41,04 \\
\hline 10 a 20 & 4,93 & 7,48 & 0,15 & 1,02 & 0,57 & 0,63 & 3,67 & 1,80 & 5,47 & 32,71 \\
\hline
\end{tabular}

${ }^{1}$ Prof. - profundidade, $\mathrm{pH}$ - potencial hidrogeniônico, $\mathrm{H}+\mathrm{Al}$ - acidez potencial, $\mathrm{S}$ - soma de bases, $\mathrm{T}$ - capacidade de troca catiônica a pH 7,0, $\mathrm{V}$ - percentagem por saturação de bases. Análises realizadas no Laboratório de Gênese e Classificação de Solos - UFRRJ.
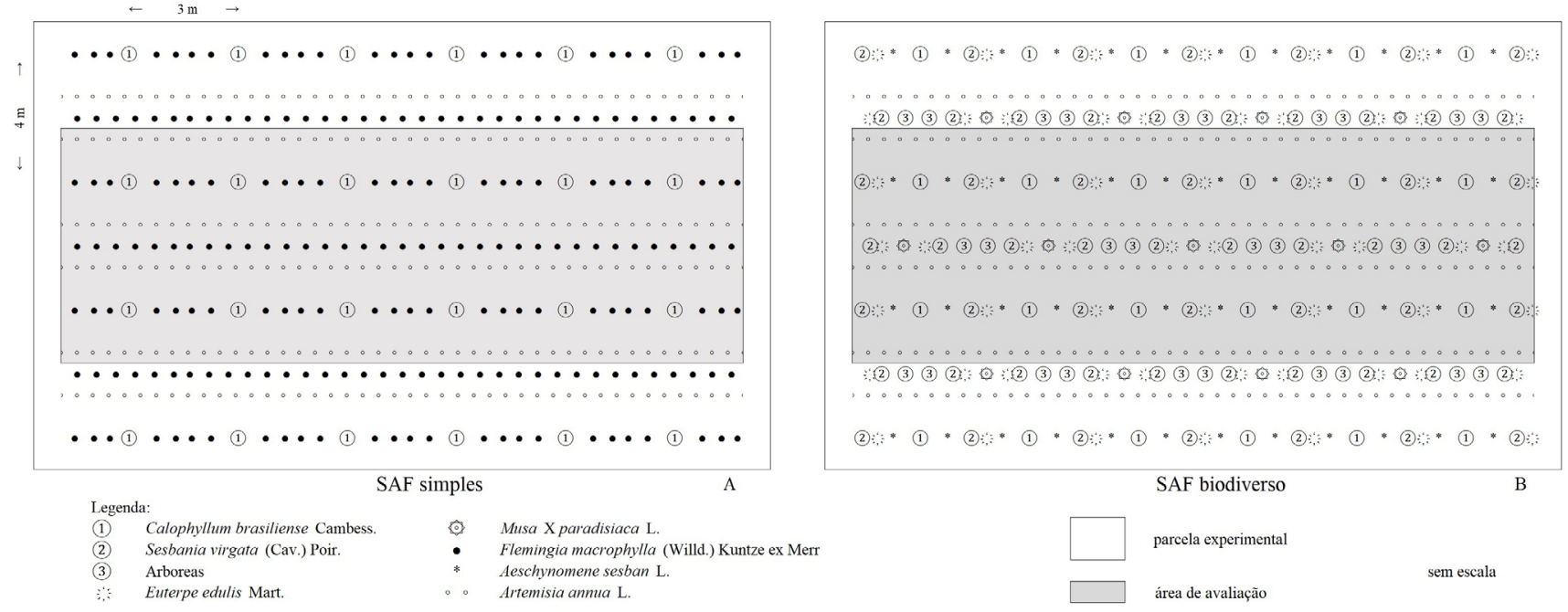

Figura 1. Desenhos experimentais dos arranjos de espécies nos sistemas agroflorestais simples (A) e biodiverso (B) em planície inundável, em Pindamonhangaba, SP.

Figure 1. Experimental designs of species arrangements in simple (A) and biodiverse (B) agroforestry systems in floodplain soils, in Pindamonhangaba, São Paulo State Brazil.

No SAF simples, as entrelinhas do guanandi (Figura 1A) foram cultivadas com duas fileiras de culturas anuais: no $1^{\circ}$ ano com artemísia (Artemisia annua L.) e nos anos subsequentes em rotação com taro (Colocasia esculenta (L.) Schott). Em consórcio com taro plantouse flemíngia (Flemingia macrophylla (Willd.) Merril), uma espécie leguminosa arbustiva manejada com podas para adubação verde. O SAF biodiverso (Figura 1B) foi enriquecido nas entrelinhas da cultura anual com mudas de bananeira (Musa sp.) e espécies arbóreas nativas: 10 espécies florestais da classe sucessional pioneira [anjico preto - Anadenanthera colubrina (Vell.) Brenan, urucum - Bixa orellana L., sangra d'água - Croton floribundus Spreng, pau viola - Citharexylum myrianthum Cham., 
eritrina - Erythrina verna Vell, ingá - Inga vera Willd., boleiro - Joannesia princeps Vell., guapuruvú Schizolobium parahyba (Vell.) Blake, sesbânia - Sesbania virgata (Cav.) Pers. e aroeira - Schinus terebinthifolius Raddi.]; e seis não pioneiras [paineira - Ceiba speciosa (A. St.-Hil.) Ravenna, juçara - Euterpe edulis Mart., ipê-rosa - Handroanthus impetiginosus (Mart. ex DC.) Mattos, ipê amarelo do brejo - Handroanthus umbellatus (Sond.) Mattos, magnólia - Magnolia ovata (A.St.-Hil.) Spreng. e imbirussú - Pseudobombax grandiflorum (Cav.) A.Robyns]. As arbóreas foram espaçadas 1,5 $m$ entre si e alternadas com sesbânia e juçara, que também foram plantadas na linha entre as árvores de guanandi e intercaladas com a arbustiva nativa paquinha (Aeschynomene sp.). O manejo de poda para adubação verde nesse sistema foi realizado em sesbânia e paquinha.

Foram avaliadas sempre as mesmas cinco árvores de guanandi, selecionadas ao acaso nas duas fileiras centrais de cada parcela de cada tratamento, desprezando-se os indivíduos das cabeceiras. A avaliação foi realizada com trena e fita métrica descartável, para altura total a partir do nível do solo até o topo $(\mathrm{H})$; raio da copa (Rcopa), com base na média de duas medidas perpendiculares tomadas na linha de cultivo a partir do tronco até a projeção lateral da copa; a circunferência à altura do solo (CAS) e a circunferência a $1,30 \mathrm{~m}$ do solo (CAP), em 26/09/2011, 18/04/2012, 22/05/2013 e 13/05/2014. A taxa de crescimento relativo (TCR) utilizada para mensurar o crescimento de espécies florestais (Antúnez et al., 2001; Melotto et al., 2009; Pioto et al., 2010; Schneider et al., 2014), foi aplicada à média dos parâmetros morfológicos das cinco árvores de guanandi de cada parcela (Equação 1), e abrangeu a diferença entre o início e o término das avaliações no intervalo de 27 meses.

$$
T C R=[(M f / M i) / \Delta t-0.05] * 100
$$

Em que: $\mathrm{Mf}=$ valor final, $\mathrm{Mi}=$ valor inicial, $\Delta \mathrm{t}=$ intervalo em meses entre as duas avaliações. A análise do crescimento do guanandi e a TCR foi utilizado o Teste de Tukey $(p<0,05)$ (UFSCAR, 2003).

\section{Resultados}

O estudo de conversão do plantio homogêneo de guanandi para sistemas agroflorestais foi iniciado quando as árvores apresentavam quatro anos e seis meses de idade, com valores médios de 3,13 $\mathrm{m}$ de altura até o topo e raio da copa de $40 \mathrm{~cm}$. O aumento na densidade de espécies nos SAFs simples e biodiverso (Tabela 2) modificou o ambiente, em comparação ao plantio homogêneo em anos de cultivo com excesso de chuvas (Figura 2) e de seca extrema (Figura 3).

Tabela 2. Densidade de espécies em plantio homogêneo de guanandi e sistemas agroflorestais (SAF's) simples e biodiverso em planície inundável, em Pindamonhangaba, SP.

Table 2. Species density in homogeneous guanandi planting and in simple and biodiverse agroforestry systems in a floodplain soil, in Pindamonhangaba, São Paulo State, Brazil.

\begin{tabular}{|c|c|c|c|}
\hline \multirow{2}{*}{ Nome científico } & \multirow{2}{*}{$\begin{array}{c}\text { Plantio } \\
\text { homogêneo }\end{array}$} & \multicolumn{2}{|c|}{ SAF } \\
\hline & & simples & biodiverso \\
\hline & \multicolumn{3}{|c|}{--------- plantas ha" } \\
\hline $\begin{array}{c}\text { Calophyllum brasiliense } \\
\text { Cambess. }\end{array}$ & 1.111 & 1.111 & 1.111 \\
\hline Artemisia anпиа $\mathrm{L}$. & - & 10.000 & 10.000 \\
\hline Colocasia esculenta (L.) Schott & - & 11.111 & 11.111 \\
\hline Aeschynomene L. & - & - & 2.222 \\
\hline $\begin{array}{l}\text { Flemingia macrophylla } \\
\text { (Willd.) Merr. }\end{array}$ & - & 12.962 & - \\
\hline Musa sp. & - & - & 833 \\
\hline Euterpe edulis Mart. & - & - & 1.944 \\
\hline Sesbania virgata (Cav.) Pers. & - & - & 3.777 \\
\hline Bixa orellana $\mathrm{L}$. & - & - & 138 \\
\hline Schinus terebinthifolius Raddi & - & - & 138 \\
\hline $\begin{array}{c}\text { Pseudobombax grandiflorum } \\
\text { (Cav.) A.Robyns }\end{array}$ & - & - & 92 \\
\hline $\begin{array}{l}\text { Anadenanthera colubrina } \\
\text { (Vell.) Brenan var. colubrina }\end{array}$ & - & - & 92 \\
\hline Inga vera Willd. & - & - & 92 \\
\hline Erythrina verna Vell. & - & - & 92 \\
\hline Joannesia princeps Vell. & - & - & 92 \\
\hline $\begin{array}{l}\text { Schizolobium parahyba (Vell.) } \\
\text { Blake }\end{array}$ & - & - & 92 \\
\hline $\begin{array}{c}\text { Ceiba speciosa (A.St.-Hil.) } \\
\text { Ravenna }\end{array}$ & - & - & 92 \\
\hline $\begin{array}{l}\text { Handroanthus impetiginosus } \\
\text { (Mart. ex DC.) Mattos }\end{array}$ & - & - & 92 \\
\hline $\begin{array}{c}\text { Cytharexylum myrianthun } \\
\text { Cham. }\end{array}$ & - & - & 92 \\
\hline Croton floribundus Spreng. & - & - & 92 \\
\hline $\begin{array}{l}\text { Handroanthus umbellatus } \\
\text { (Sond.) Mattos }\end{array}$ & - & - & 46 \\
\hline $\begin{array}{l}\text { Magnolia ovata (A.St.-Hil.) } \\
\text { Spreng. }\end{array}$ & - & - & 46 \\
\hline
\end{tabular}



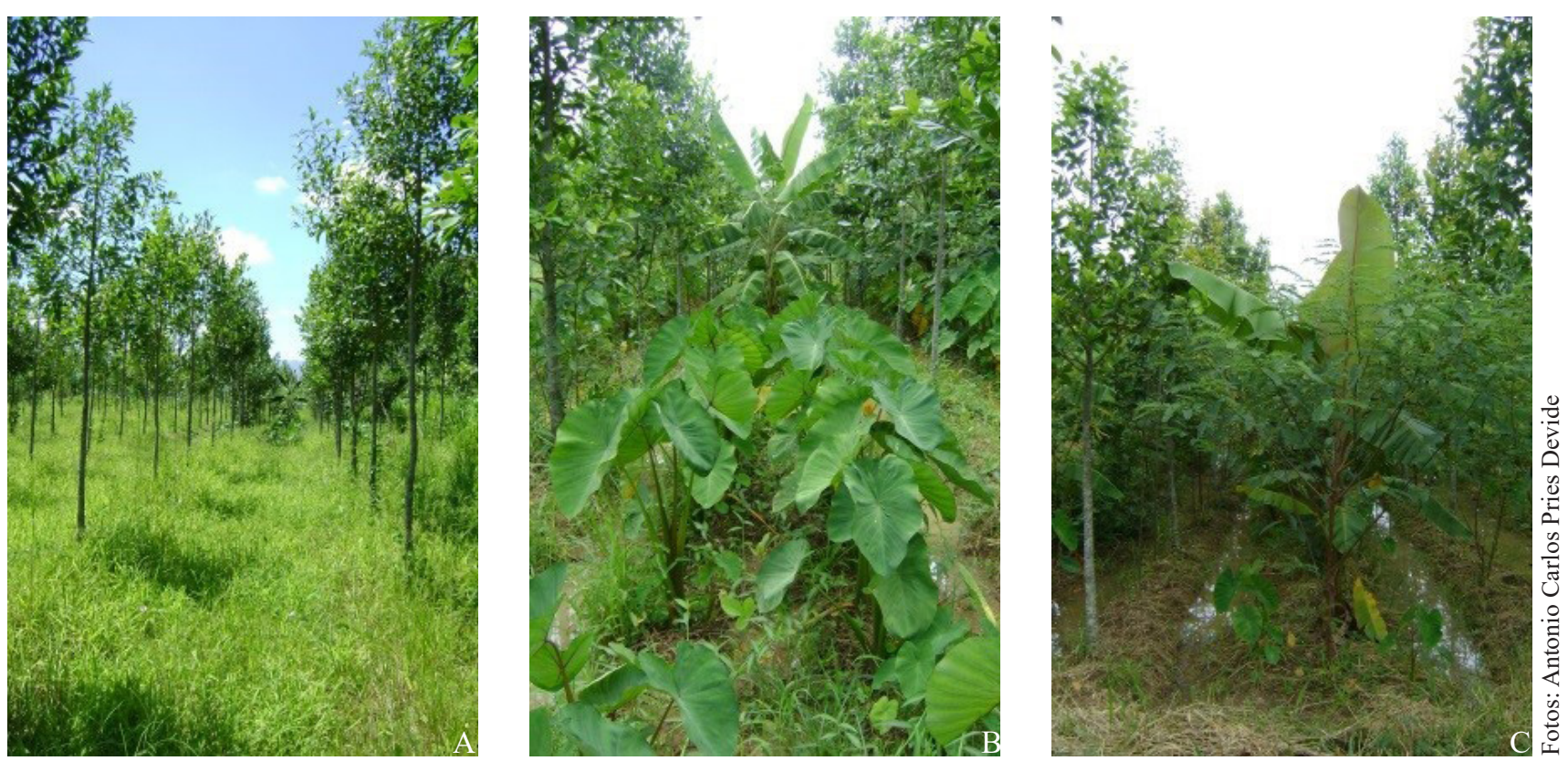

Figura 2. Plantio de guanandi (2012) em (A) talhão homogêneo, (B) sistema agroflorestal (SAF) simples e (C) SAF Biodiverso, em planície inundável, em Pindamonhangaba, SP.

Figure 2. Guanandi planting (2012), as (A) homogeneous stand, (B) Simple agroforestry system (AFS) and (C) biodiverse AFS, in a floodplain soil, in Pindamonhangaba, São Paulo State.
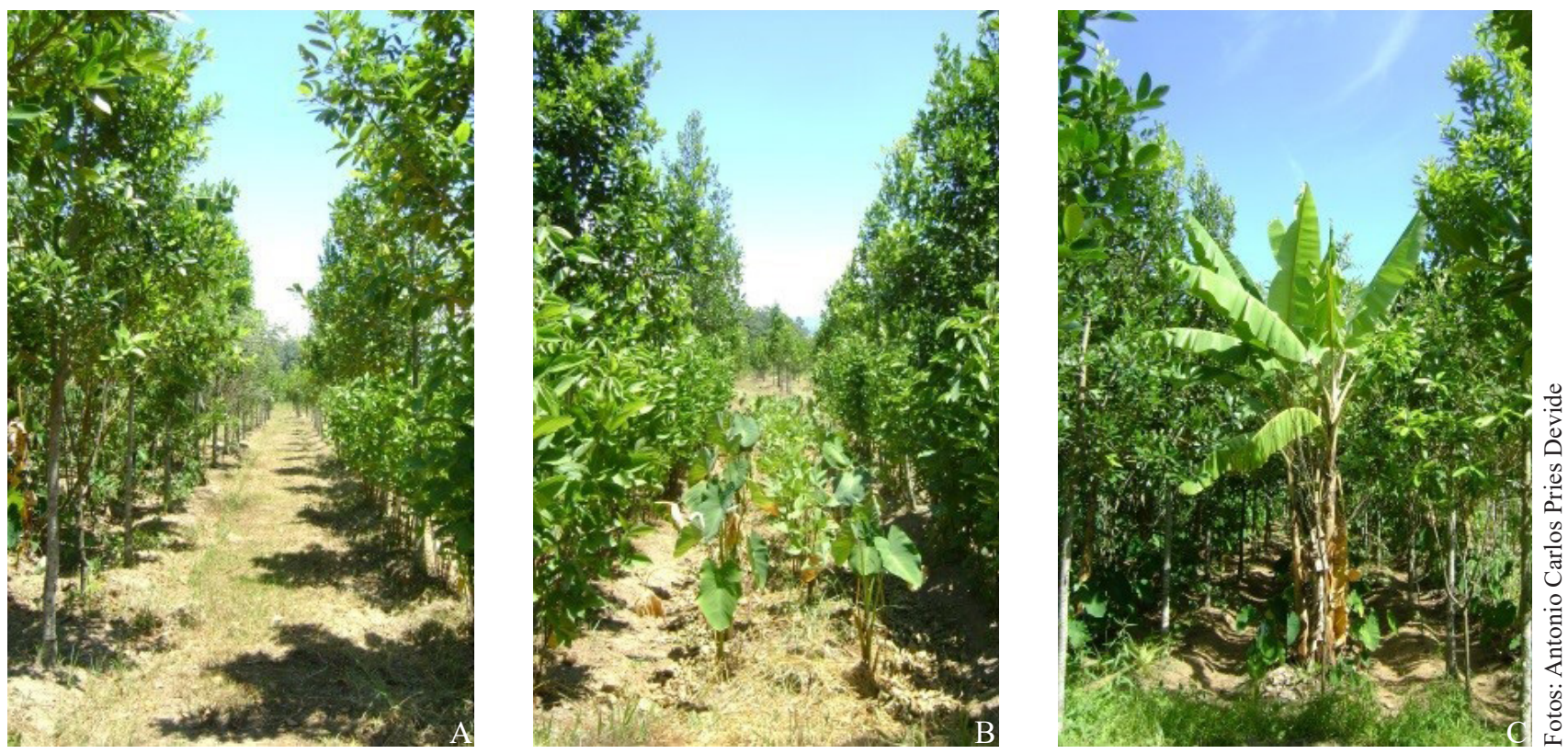

Figura 3. Plantio de guanandi (2014) em (A) talhão homogêneo, (B) sistema agroflorestal (SAF) simples e (C) SAF Biodiverso, em planície inundável na seca, em Pindamonhangaba, SP.

Figure 3. Guanandi planting (2014), as (A) homogeneous stand, (B) Simple agroforestry system (AFS) and (C) biodiverse AFS, in a floodplain soil in drought, in Pindamonhangaba, São Paulo State. 
O guanandi apresentou diferenças morfológicas não significativas entre o plantio homogêneo e os sistemas agroflorestais simples e biodiverso em planície aluvial, com exceção apenas para a circunferência à altura do solo (CAS) no ano de 2013 em que o valor no SAF simples foi significativamente inferior (Tabela 3). Com sete anos de idade o guanandi apresentou valores médios de 5,40 m de altura até o topo, circunferência a 1,30 m do solo (CAP) de 22,80 cm, CAS de 40,53 cm e raio de copa de $96,80 \mathrm{~cm}$.

A taxa de crescimento relativo (TCR) do guanandi não diferiu entre o plantio homogêneo e os sistemas agroflorestais simples e biodiverso (Tabela 4).

Tabela 3. Diferenças morfológicas em guanandi em plantio homogêneo e sistemas agroflorestais simples e biodiverso em planície inundável, em Pindamonhangaba, SP.

Table 3. Morphological differences in guanandi in homogeneous planting and simple and biodiversity agroforestry systems in floodplain, in Pindamonhangaba, São Paulo State, Brazil.

\begin{tabular}{|c|c|c|c|c|c|c|c|c|c|}
\hline \multirow{2}{*}{ Parâmetros } & \multirow{2}{*}{ Ano } & \multirow{2}{*}{$\begin{array}{c}\text { Plantio } \\
\text { homogêneo }\end{array}$} & \multicolumn{2}{|c|}{ SAF } & \multirow{2}{*}{ Média } & \multirow{2}{*}{$\begin{array}{l}\text { Desvio- } \\
\text { padrão }\end{array}$} & \multirow{2}{*}{$\begin{array}{l}\text { DMS } \\
(5 \%)\end{array}$} & \multirow{2}{*}{ CV (\%) } & \multirow{2}{*}{ F tratamentos } \\
\hline & & & simples & biodiverso & & & & & \\
\hline \multirow{4}{*}{$\begin{array}{c}\text { Altura } \\
(\mathrm{m})\end{array}$} & 2011 & $3,19 \mathrm{a}$ & $3,14 \mathrm{a}$ & $3,07 \mathrm{a}$ & 3,13 & 0,25 & 0,32 & 7,87 & $0,41^{\mathrm{ns}}$ \\
\hline & 2012 & $3,65 \mathrm{a}$ & $3,65 \mathrm{a}$ & $3,56 \mathrm{a}$ & 3,62 & 0,30 & 0,39 & 8,29 & $0,24{ }^{\mathrm{ns}}$ \\
\hline & 2013 & $4,53 \mathrm{a}$ & $4,57 \mathrm{a}$ & $4,35 \mathrm{a}$ & 4,48 & 0,47 & 0,62 & 10,50 & $0,47^{\mathrm{ns}}$ \\
\hline & 2014 & $5,33 \mathrm{a}$ & $5,45 \mathrm{a}$ & $5,42 \mathrm{a}$ & 5,40 & 0,55 & 0,72 & 10,25 & $0,11^{\text {ns }}$ \\
\hline \multirow{4}{*}{$\begin{array}{l}\text { Raio da copa } \\
\qquad(\mathrm{cm})\end{array}$} & 2011 & $41,79 \mathrm{a}$ & $39,28 \mathrm{a}$ & 38,86 a & 39,98 & 4,87 & 6,37 & 12,19 & $0,85^{\mathrm{ns}}$ \\
\hline & 2012 & 57,09 a & $55,23 \mathrm{a}$ & 54,08 a & 55,46 & 8,03 & 10,51 & 14,48 & $0,29^{\text {ns }}$ \\
\hline & 2013 & $79,34 \mathrm{a}$ & $77,55 \mathrm{a}$ & 72,76 a & 76,55 & 12,44 & 16,27 & 16,25 & 0,60 ns \\
\hline & 2014 & $102,41 \mathrm{a}$ & $93,39 \mathrm{a}$ & 94,61 a & 96,80 & 17,75 & 23,23 & 18,34 & 0,61 ns \\
\hline \multirow{4}{*}{$\begin{array}{l}\text { CAS } \\
(\mathrm{cm})\end{array}$} & 2011 & $22,52 \mathrm{a}$ & $22,59 \mathrm{a}$ & $21,82 \mathrm{a}$ & 22,31 & 1,67 & 2,18 & 7,47 & $0,52^{\text {ns }}$ \\
\hline & 2012 & $26,30 \mathrm{a}$ & $26,52 \mathrm{a}$ & $25,68 \mathrm{a}$ & 26,17 & 2,04 & 2,67 & 7,80 & $0,36^{\mathrm{ns}}$ \\
\hline & 2013 & $35,79 \mathrm{a}$ & $28,96 \mathrm{~b}$ & $34,42 \mathrm{a}$ & 33,06 & 3,11 & 4,07 & 9,41 & $10,79^{* *}$ \\
\hline & 2014 & 39,89 a & $41,86 \mathrm{a}$ & $39,85 \mathrm{a}$ & 40,53 & 5,55 & 7,26 & 13,69 & $0,34^{\mathrm{ns}}$ \\
\hline \multirow{4}{*}{$\begin{array}{l}\text { CAP } \\
(\mathrm{cm})\end{array}$} & 2011 & $10,55 \mathrm{a}$ & $10,79 \mathrm{a}$ & 10,03 a & 10,46 & 1,65 & 2,17 & 15,82 & $0,45^{\mathrm{ns}}$ \\
\hline & 2012 & $13,64 \mathrm{a}$ & $13,76 \mathrm{a}$ & $13,18 \mathrm{a}$ & 13,53 & 1,82 & 2,38 & 13,45 & 0,22 ns \\
\hline & 2013 & 20,09 a & $25,51 \mathrm{a}$ & $19,40 \mathrm{a}$ & 21,66 & 6,65 & 8,70 & 30,67 & $2,03^{\mathrm{ns}}$ \\
\hline & 2014 & $22,05 \mathrm{a}$ & $23,70 \mathrm{a}$ & $22,65 \mathrm{a}$ & 22,80 & 3,96 & 5,19 & 17,39 & $0,36^{\mathrm{ns}}$ \\
\hline
\end{tabular}

Teste de Tukey - Nível de significância: **: 1\%; *:5\%; DMS - diferença mínima significativa; CV - coeficiente de variação; CAS - circunferência à altura do solo; CAP - circunferência a 1,30 m de altura; SAF - sistema agroflorestal. 
Tabela 4. Taxa de crescimento relativo (TCR) de guanandi em plantio homogêneo e sistemas agroflorestais simples e biodiverso em planície inundável, em Pindamonhangaba, SP.

Table 4. Guanandi relative growth rate (TCR) in homogeneous planting and simple and biodiverse agroforestry systems in floodplain, in Pindamonhangaba, São Paulo State, Brazil.

\begin{tabular}{ccccc}
\hline Sistemas & $\begin{array}{c}\text { Altura } \\
(\mathbf{m})\end{array}$ & $\begin{array}{c}\text { Raio da } \\
\mathbf{c o p a} \\
(\mathbf{m})\end{array}$ & $\begin{array}{c}\text { CAS } \\
\mathbf{( c m )}\end{array}$ & $\begin{array}{c}\text { CAP } \\
\mathbf{( c m )}\end{array}$ \\
\hline $\begin{array}{c}\text { Plantio } \\
\text { homogêneo }\end{array}$ & $0,24 \mathrm{a}$ & $2,68 \mathrm{a}$ & $0,54 \mathrm{a}$ & $1,57 \mathrm{a}$ \\
SAF simples & $0,41 \mathrm{a}$ & $2,42 \mathrm{a}$ & $0,81 \mathrm{a}$ & $1,90 \mathrm{a}$ \\
SAF biodiverso & $0,53 \mathrm{a}$ & $2,68 \mathrm{a}$ & $0,85 \mathrm{a}$ & $2,11 \mathrm{a}$ \\
F tratamentos & 1,05 ns & $0,16^{\mathrm{ns}}$ & $0,51 \mathrm{~ns}$ & $0,72 \mathrm{~ns}$ \\
Média geral & 0,39 & 2,59 & 0,73 & 1,86 \\
Desvio-padrão & 0,41 & 1,07 & 0,67 & 0,90 \\
DMS (5\%) & 0,53 & 1,40 & 0,88 & 1,18 \\
CV (\%) & 103,14 & 41,39 & 92,06 & 48,57 \\
\hline
\end{tabular}

Teste de Tukey - Nível de significância: **: 1\%; *: 5\%; DMS - diferença mínima significativa; CV - coeficiente de variação; CAS - circunferência à altura do solo; CAP - circunferência a $1,30 \mathrm{~m}$ de altura; $\mathrm{SAF}$ - sistema agroflorestal.

\section{Discussão}

O formato da parte aérea do guanandi, de piramidal nos primeiros anos de vida a ovoide no estágio seguinte, e o moderado raio da copa no início do experimento favoreceram a associação de culturas agrícolas, adubos verdes e outras espécies arbóreas nativas nas entrelinhas (Figuras 2 e 3). Isto melhorou a cobertura do solo e o uso econômico em curto, médio e longo prazos, enquanto que no plantio homogêneo houve demanda frequente de controle da vegetação herbácea, principalmente de espécies das famílias Poaceae e Cyperaceae.

O crescimento do guanandi até aos sete anos de idade em planície aluvial foi similar entre os sistemas avaliados. Esses dados estão de acordo com os registros dessa espécie na Costa Rica (Petit \& Montagnini, 2006), nas Filipinas (Redondo-Brenes \& Montagnini, 2006) e no Brasil (Carvalho, 2003; Devide et al., 2019). Em Manaus, AM, árvores de guanandi com a mesma idade apresentaram altura média de $4,68 \mathrm{~m}$ e $12 \mathrm{~cm}$ de circunferência a $1,30 \mathrm{~m}$ de altura (CAP), ao passo que em Paranaguá, PR, Carvalho (2003) registrou árvores de até $7,81 \mathrm{~m}$ de altura e $31 \mathrm{~cm}$ de CAP. Em Pindamonhangaba, SP, Devide et al. (2019) relataram que em terraço fluvial com boa fertilidade natural, o guanandi apresentou $6,17 \mathrm{~m}$ de altura com sete anos e CAP significativamente superior quando em SAF simples $(27,2 \mathrm{~cm})$, em comparação ao plantio homogêneo $(24,0 \mathrm{~cm})$.

Embora o guanandi seja classificado como tolerante à inundação do solo (Carvalho, 2003; Redondo-Brenes \& Montagnini, 2006; Oliveira \& Joly, 2010; Durigan et al., 2012) e as plantas continuarem a incorporar carbono nessa condição, ocorre redução na concentração total de clorofila e na taxa de assimilação de dióxido de carbono quando em ambiente inundado (Oliveira \& Joly, 2010). $\mathrm{O}$ alagamento modifica os fluxos de solutos e gases e reduz o teor de oxigênio do solo (Pezeshki \& Delaune, 2010). Com isso, o guanandi precisa canalizar mais fotoassimilados para as transformações celulares, como a hipertrofia de lenticelas e o desenvolvimento de raízes adventícias, que por sua vez possibilitam a sobrevivência em áreas alagadas (Oliveira \& Joly, 2010). Dessa forma, as diferenças de crescimento do guanandi, em terraço fluvial (Devide et al., 2019) e planície inundável parecem estar associadas, principalmente, às condições da drenagem do solo.

Nessa pesquisa, as taxas de crescimento relativo (TCR) obtidas com alternância de períodos de chuvas intensas nos dois primeiros anos (média de $1.402 \mathrm{~mm}$ ), que causaram a inundação dos solos, com períodos extremamente secos nos dois anos seguintes (média $889 \mathrm{~mm}$ ) em uma magnitude até então ainda não registrada no Vale do Paraíba do Sul (Targa \& Batista, 2015), foram similares entre o plantio homogêneo e os sistemas agroflorestais. A TCR ocorre em função de um componente morfológico, a razão de área foliar (RAF), que é a área foliar por unidade de massa seca total, e de um componente fisiológico, a taxa de assimilação líquida (TAL) (Pinzon-Torres \& Schiavinato, 2008). Para Antúnez et al. (2001) na maioria das vezes as variações na TCR de espécies florestais são atribuídas à área foliar. Depreende-se que os consórcios não influenciaram as variáveis fisiológicas do guanandi, uma vez que as variáveis morfológicas das árvores apresentaram distribuição dos dados similares em plantio homogêneo e em sistemas agroflorestais.

Quando ocorre o fechamento do dossel, o conjunto de espécies consorciadas precisa ser manejado, com condução das espécies tolerantes à sombra e a poda. Dessa forma, para se evitar o sombreamento do guanandi, que é uma espécie secundária intermediária tardia com moderada tolerância à sombra (Schneider et al., 2014), as espécies de rápido crescimento arbustivas 
e arbóreas empregadas para adubação verde foram podadas. No sistema agroflorestal o manejo de poda visa garantir a luminosidade adequada para a(s) espécie(s) de interesse econômico e aportar fitomassa ao solo, para incremento na fertilidade. Para Benincasa (2003), as interações com o ambiente interferem na análise quantitativa do crescimento. Para esse autor, cerca de $90 \%$ são provenientes da matéria orgânica acumulada da atividade fotossintética ao longo do crescimento da planta e o restante é devido à absorção mineral do solo.

O manejo agroflorestal, semelhante ao manejo silvicultural, converte espécies regenerantes em árvores para o aproveitamento futuro, reduzindo o custo financeiro e promovendo o aumento do crescimento das espécies de interesse econômico remanescentes (Piazza et al., 2017). Esse manejo pode favorecer o crescimento inicial do guanandi e também a restauração ecológica. Além dos produtos florestais e não florestais obtidos ao longo do tempo, pode-se pleitear o pagamento por serviços ambientais (PSA) nos sistemas agroflorestais (Brancalion et al., 2012), considerando que a região de Mata Atlântica abriga a maioria dos projetos de PSA em andamento no Brasil (Guedes \& Seehusen, 2011).

A resiliência de sistemas de produção biodiversos em regiões tropicais degradadas favorece o desempenho de espécies de interesse econômico (Luedeling et al., 2014; Altieri \& Nicholls, 2017; Basche \& Edelson, 2017; FAO, 2017; Laudares et al., 2017; Devide et al., 2019). Apesar de sua importância, esses conhecimentos ainda são limitados para generalizar recomendações de enriquecimento florestal (Rappaport \& Montagnini, 2014). Pesquisas em longo prazo, que forneçam diretrizes confiáveis aos programas de reflorestamento com espécies nativas são importantes, pois para muitas espécies, como o guanandi, o crescimento e a sobrevivência juvenil nem sempre são sustentadas na maturidade (Piotto et al., 2010).

\section{Conclusão}

As plantas de guanandi apresentaram similaridade morfológica e de crescimento relativo de copa e diâmetro, quando em plantio homogêneo e em sistemas agroflorestais em planície de inundação.

Os sistemas agroflorestais com guanandi em planície de inundação são promissores, por aliarem os benefícios da geração de renda e da restauração ecológica.

\section{Agradecimentos}

Os autores expressam seus agradecimentos a: Fazenda Coruputuba, pelo custeio das pesquisas e a Fundação de Apoio a Pesquisa Agrícola (FUNDAG) pela gestão financeira do projeto; ao pesquisador José Guilherme Marinho Guerra da Embrapa Agrobiologia, pelo suporte na elaboração do desenho experimental; e ao professor Maurício Ballesteiro Pereira da Universidade Federal Rural do Rio de Janeiro (UFRRJ), pelo auxílio na análise estatística.

\section{Referências}

Altieri, M. A. \& Nicholls, C. I. The adaptation and mitigation potential of traditional agriculture in a changing climate. Climatic Change, v. 140, n. 1, p. 33-45, 2017. https://doi.org/10.1007/ s10584-013-0909-y.

Antúnez, I. et al. Relative growth rate in phylogenetically related deciduous and evergreen woody species. Oecologia, v. 128, p. 172, 2001. https://doi.org/10.1007/s004420100645.

Attanasio, C. M. et al. Métodos para restauração de florestas de brejo degradadas. Pesquisa \& Tecnologia, v. 12, n. 1, 2015.

Baptista, M. N. \& Valcarcel, R. Renaturalizing floodplains. Journal of Water Resource and Protection, v. 10, p. 533-537, 2018. https:// doi.org/10.4236/jwarp.2018.105029.

Basche, A. D. \& Edelson, O. F. Improving water resilience with more perennially based agriculture. Agroecology and Sustainable Food Systems, v. 41, n. 7, p. 799-824, 2017. https://doi.org/10.108 0/21683565.2017.1330795.

Benincasa, M. M. P. Análise de crescimento de plantas: noções básicas. Jaboticabal: FUNEP. 2003. 42 p.

Bittencourt, L. F. F. \& Batista, G. T. Intervenção humana na mata ciliar do Rio Paraíba do Sul, município de Caçapava. Revista OKARA: Geografia em Debate, v. 3, n. 2, p. 223-347, 2009.

Brancalion, P. H. S. et al. Estratégias para auxiliar na conservação de florestas tropicais secundárias inseridas em paisagens alteradas. Boletim do Museu Paraense Emílio Goeldi. Ciências Naturais, v. 7, n. 3, p. 219-234, 2012.

Carvalho, P. E. R. Guanandi. Colombo: Embrapa Florestas, 2003. 14 p. Embrapa Florestas. Circular técnica, 78). http://ainfo.cnptia. embrapa.br/digital/bitstream/item/163849/1/CT-78-Paulo-ErnaniCarvalho.pdf.

Coelho, R. C. T. P. et al. Influência do uso e ocupação do solo na qualidade da água: um método para avaliar a importância da zona ripária. Ambiente \& Água, v. 6, n. 1, p. 104-117, 2011. https://doi. org/10.4136/ambi-agua.177.

Coutinho, M. P. et al. Áreas de inundação no trecho paulista da bacia do Rio Paraíba do Sul e nascentes do Cadastro Ambiental Rural. Urbe, Revista Brasileira de Gestão Urbana, v. 10, n. 3, p. 614-623, 2018. https://doi.org/10.1590/2175-3369.010.003.ao09. 
Del-Rio, G. et al. Multiscale approach indicates a severe reduction in Atlantic Forest Wetlands and Highlights that São Paulo Marsh Antwren is on the brink of extinction. PLoS ONE, v. 10, n. 3, e0121315, 2015. https://doi.org/10.1371/journal.pone.0121315.

Devide, A. C. P. et al. Crescimento do guanandi e produção de mandioca e araruta em sistemas agroflorestais. Revista Verde de Agroecologia e Desenvolvimento Sustentável, v. 14, n. 2, p. 303-311, 2019. https://doi.org/10.18378/rvads.v14i2.6306.

Donagema, G. K. et al. (org.) Manual de métodos de análise de solos. 2. ed. rev. Rio de Janeiro: Embrapa Solos, 2011. 230 p. (Embrapa Solos. Documentos, 132).

D’Orazio, F. A. E. \& Catharino, E. L. M. Estrutura e florística de dois fragmentos de florestas aluviais no Vale do rio Paraíba do Sul, SP, Brasil. Hoehnea, v. 40, n. 3, p. 567-582, 2013. https://doi. org/10.1590/S2236-89062013000300015.

Durigan, G. et al. Espécies indicadoras de fitofisionomias na transição cerrado-mata atlântica no estado de São Paulo. São Paulo: Secretaria do Meio Ambiente, 2012. 146 p.

FAO. Agroforestry for landscape restoration: exploring the potential of agroforestry to enhance the sustainability and resilience of degraded landscapes. Roma: FAO, 2017. 28 p.

Friday, J. B. \& Ogoshi, R. Farm and forestry production and marketing profile for Tamanu (Calophyllum inophyllun). In: Elevitch, C. R. (ed.). Specialty crops for Pacific Island Agroforestry. Holualoa: Agroforestry Net, 2011. 13 p. http://agroforestry.net/scps/ Tamanu_specialty_crop.pdf.

Friday, J. B. \& Okano, D. Calophyllum inophyllum (kamani). In: Elevitch, C. R. (ed.). Species profiles for Pacific Island Agroforestry. Holualoa: Agroforestry Net, 2006. 17 p. http:// agroforestry.net/tti/Calophyllum-kamani. pdf.

Guedes, F. M. \& Seehusen, S. E. (org.). Pagamento por serviços ambientais na Mata Atlântica: lições aprendidas e desafios. Brasília, DF: Ministério do Meio Ambiente, 2011. 272 p.

Laudares, S. S. A. et al. Agroforestry as a sustainable alternative for environmental regularization of rural consolidated occupations. Cerne, v. 23, n. 2, p. 161-174, 2017. https://doi.org/10.1590/0104 7760201723022240 .

Luedeling, E. et al. Agroforestry systems in a changing climate: challenges in projecting future performance. Environmental Sustainability, v. 6, p. 1-7, 2014. https://doi.org/10.1016/j. cosust.2013.07.013.

Melotto, A. et al. Sobrevivência e crescimento inicial em campo de espécies florestais nativas do Brasil Central indicadas para sistemas silvipastoris. Revista Árvore, v. 33, n. 3, p. 425-432, 2009. https:// doi.org/10.1590/S0100-67622009000300004.

Minnemeyer, S. et al. Atlas of forest landscape restoration opportunities. Washington, DC: World Resources Institute, International Union for Conservation of Nature and South Dakota State University, 2011. http://www. wri.org/resources/maps/globalmap-forest-landscape-restoration-opportunities.
Oliveira, V. C. \& Joly, C. A. Flooding tolerance of Calophyllum brasiliense Camb. (Clusiaceae): morphological, physiological and growth responses. Trees, v. 24, p. 185-193, 2010. https://doi. org/10.1007/s00468-009-0392-2.

Petit, B. \& Montagnini, F. Growth in pure and mixed plantations of tree species used in reforesting rural areas of the humid region of Costa Rica, Central America. Forest Ecology and Management, v. 233, p. 338-343, 2006. https://doi.org/10.1016/j.foreco.2006.05.030.

Pezeshki, S. R. \& Delaune, R. D. Soil oxidation-reduction in wetlands and its impact on plant functioning. Biology, v. 1, p. 196-221, 2010. https://doi.org/10.3390/biology1020196.

Piazza, G. E. et al. Regeneração natural de espécies madeireiras na floresta secundária da Mata Atlântica. Advances in Forestry Science, v. 4, n. 2, p. 99-105, 2017.

Pinzon-Torres, J. A. \& Schiavinato, M. A. Eficiência de crescimento, fotossíntese e uso da água em quatro espécies de leguminosas tropicais. Hoehnea, v. 35, n. 3, p. 395-404, 2008. https://doi. org/10.1590/S2236-89062008000300007.

Piotto, D. et al. Silvicultural and economic aspects of pure and mixed native tree species plantations on degraded pasturelands in humid Costa Rica. New Forests, v. 39, p. 369-385, 2010. https:// doi.org/10.1007/s11056-009-9177-0.

Rappaport, D. \& Montagnini, F. Tree species growth under a rubber (Hevea brasiliensis) plantation: native restoration via enrichment planting in southern Bahia, Brazil. New Forests, v. 45, n. 5, p. 715732, 2014. https://doi.org/10.1007/s11056-014-9433-9.

Redondo-Brenes, A. \& Montagnini, F. Growth, productivity, aboveground biomass, and carbon sequestration of pure and mixed native tree plantations in the Caribbean lowlands of Costa Rica. Forest Ecology and Management, v. 232, n. 1/3, p. 168-178, 2006. https://doi.org/10.1016/j.foreco.2006.05.067.

Santos, H. G. et al. Sistema brasileiro de classificação de solos. 3 . ed. Brasília, DF: Embrapa, 2013. 353 p.

Schneider, T. et al. Growth performance of sixty tree species in smallholder reforestation trials on Leyte, Philippines. New Forests, v. 45, n. 1, p. 83-96, 2014. https://doi.org/10.1007/s11056-0139393-5.

Schroth, G. et al. Conversion of secondary forest into agroforestry and monoculture plantations in Amazonia: consequences for biomass, litter and soil carbon stocks after 7 years. Forest Ecology and Management, v. 163, p. 131-150, 2002. https://doi.org/10.1016/ S0378-1127(01)00537-0.

Souza, A. F. et al. Desenvolvimento inicial e eficiência de uso de água e nitrogênio por mudas de Calophyllum brasiliense, Eucalyptus urograndis, Tabebuia impetiginosa e Toona ciliata. Ciência Florestal, v. 28, n. 4, p. 1465-1477, 2018. https://doi. org/10.5902/1980509835054.

Targa, M. S. \& Batista, G. T. Benefits and legacy of the water crisis in Brazil. Revista Ambiente \& Água, v. 10, n. 2, p. 234-239, 2015. http://dx.doi.org/10.4136/ambi-agua.1629.

UFSCAR. Universidade Federal de São Carlos. Centro de Ciências Agrárias. Teste de Tukey. São Carlos, 2009. Disponível em: https:// www.cca.ufscar.br/pt-br/servicos/teste-de-tukey. Acesso em: 01 maio 2015 . 